\section{Digital dental photography. Part 5: lighting}

\section{Ahmad'}

IN BRIEF
- Choosing the appropriate type of lighting
is essential for obtaining a correctly
exposed, high quality, detailed image.
- The ideal lighting for dental photography
are camera mounted electronic flashes.
- By using reflectors and diffusers, light
can be modified for specific applications,
or for conveying a particular mood or
ambiance.

Effective lighting is the key to success in all photography but particularly in dental photography. This part of the series on digital dental photography examines the colour/space/time triad which is achieved by the presence and manipulation of light, be it daylight, flashlight or other forms of illumination. In addition, the effect of reflectors is described as well as their effects on colour temperature.

It is often stated that photography is the magic by which light is transformed in colour, space and time. This description is congruous to human visual experience, which is also divided into these three categories. Since light is the protagonist in both photography and visual perception, it is logical to commence with a discussion about its properties.

Dental photography encompasses three facets of the visual experience, colour, space and time:

- Colour is a three dimensional entity consisting of hue, value and chroma

\section{FUNDAMENTALS OF DIGITAL DENTAL PHOTOGRAPHY}

1. Digital dental photography: an overview

2. Purposes and uses

3. Principles of digital photography

4. Choosing a camera and accessories

5. Lighting

6. Camera settings

7. Extra-oral set-ups

8. Intra-oral set-ups

9. Post-image capture processing

10. Printing, publishing and presentations

'General Dental Practitioner, The Ridgeway Dental Surgery, 173 The Ridgeway, North Harrow, Middlesex, HA2 7DF

Correspondence to: Irfan Ahmad

Email: iahmadbds@aol.com www.IrfanAhmedTRDS.co.uk

\section{Refereed Paper}

Accepted 15 November 2008

DOI: $10.1038 /$ sj.bdj.2009.558

${ }^{\circledR}$ British Dental Journal 2009; 207: 13-18
- Space defines depth, transparency, size, shape/form and texture

- Time indicates movement, flicker, sparkle (vitality), fluctuation and glitter.

In dentistry the pink gingival colour indicates health, correct tooth proportion (shape) conveys pleasing aesthetics, and a smile infers a pleasurable moment in time.

\section{CHARACTERISTICS OF LIGHT}

The colour/space/time triad is achieved by the presence and manipulation of light. Radiant energy (light) is a narrow band of the electromagnetic spectrum, which ranges from long waves of radio broadcasting to short waves of gamma radiation.

\section{Visual and greyscale}

The visual system of the eye is capable of detecting only wavelengths ranging from $380 \mathrm{~nm}$ (violet) to $780 \mathrm{~nm}$ (red), where ' $n$ ' ' is a nanometre or one billionth of a meter. According to Isaac Newton, light has no colour, it is only when it interacts with an object that colour is produced. This was depicted in his famous experiment by splitting light using a glass prism into the colours of the visible spectrum or rainbow, which comprises red, orange, yellow, green, blue, indigo and violet (Fig. 1). The significance of Newton's experiment is that an object's colour is due to the colours it reflects, not the colours it absorbs. For example, a tomato appears red because it absorbs all the colours of the rainbow except red, which is reflected off its surface (Fig. 2). Similarly,

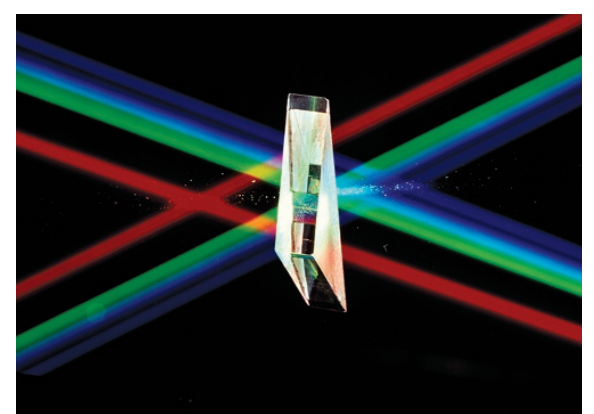

Fig. 1 Variation of the Isaac Newton experiment of splitting white light, using a prism, into the colours of the visible spectrum

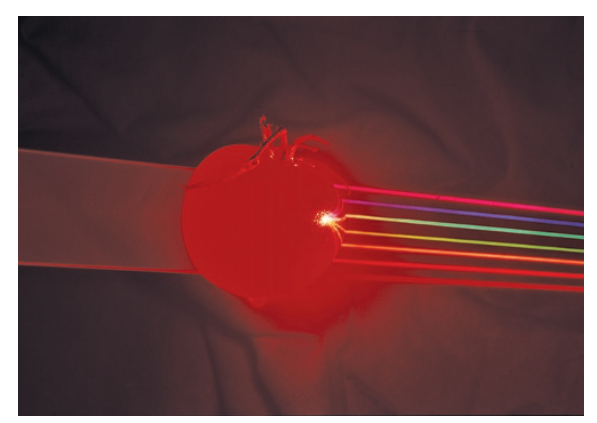

Fig. 2 A tomato appears red because it absorbs all colours except red, which is reflected off its surface

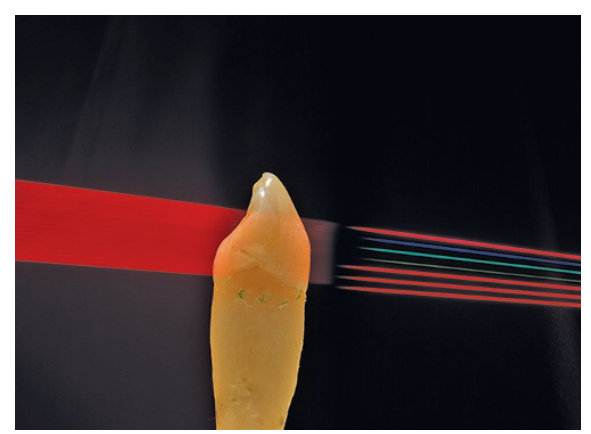

Fig. 3 A tooth appears yellow/red because it absorbs all colours except those in the yellow/ red wavelengths 
a tooth appears yellow because it absorbs all colours except those in the yellow/red wavelengths (Fig. 3).

Ideally, photography requires a continuous spectrum light source. A continuous spectrum is when all colours of the visible spectrum overlap completely, for example, natural daylight or tungsten lamps (Fig. 4). Conversely, electronic flash tubes produce a discontinuous or linear spectrum, where colours of the spectrum do not merge seamlessly but are distinct entities (Fig. 5). However, for practical purposes, this transition is negligible and can be neglected. When spectrum transitions are profound, for example, from lights that utilise gases and vapours such as fluorescent tubes, the emitted light is unsuitable for taking photographs.

Colour is composed of three dimensions, hue, value and chroma. If hue and chroma are eliminated the result is a monochromatic visual experience termed chrominance. This is, of course, the basis of black and white photography, which simply records lightness and darkness (values) or tones and gradations on a greyscale. The average of the tones on a greyscale has a value of 18\% grey (Fig. 6). The latter is known as neutral density, which is used for calibrating light meters, assessing exposure and setting the white balance of a digital image.

\section{Colour temperature}

As mentioned previously, any light source with a continuous spectrum can be used for producing photographic images. For dental photography, the choices available are natural daylight, electronic flash, tungsten incandescent bulbs (domestic or photoflood lamps), quartz lighting fixtures, HMI illumination, LED and UV.

The quality of a light source depends on its colour temperature, which is measured in Kelvin (K). The colour temperature is an indication of the quality of light; a low figure indicates a warm light (red-yellow), while a higher figure signifies a cool light (blue-white) (Fig. 7). For example, the reddish yellow light emitted by a candle matches that of a blackbody at a temperature of $1,800 \mathrm{~K}$. A blackbody is a theoretical object capable of reflecting all light falling onto its surface. In reality, most objects do not conform to this ideal, but for practical purposes the definition is acceptable. As

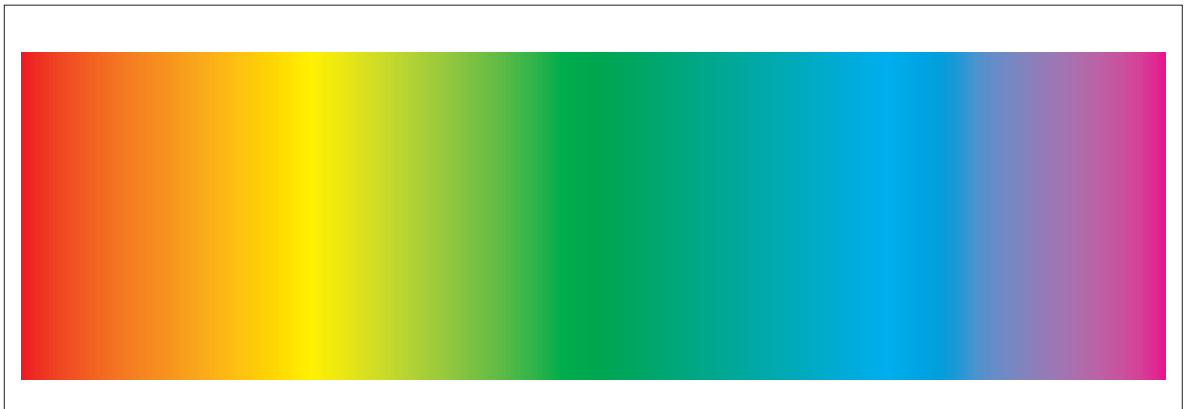

Fig. 4 Natural daylight produces a continuous, seamless spectrum

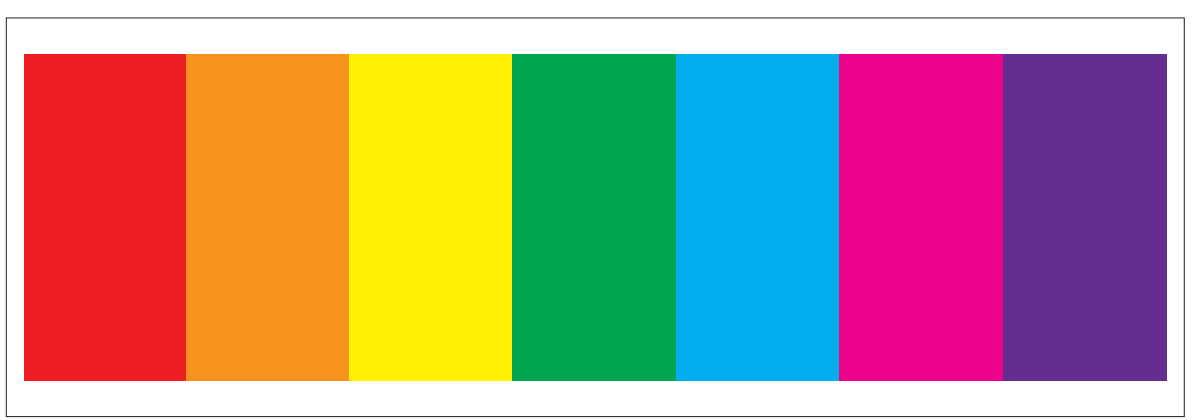

Fig. 5 Electronic flash produces a discontinuous spectrum with distinct transitions

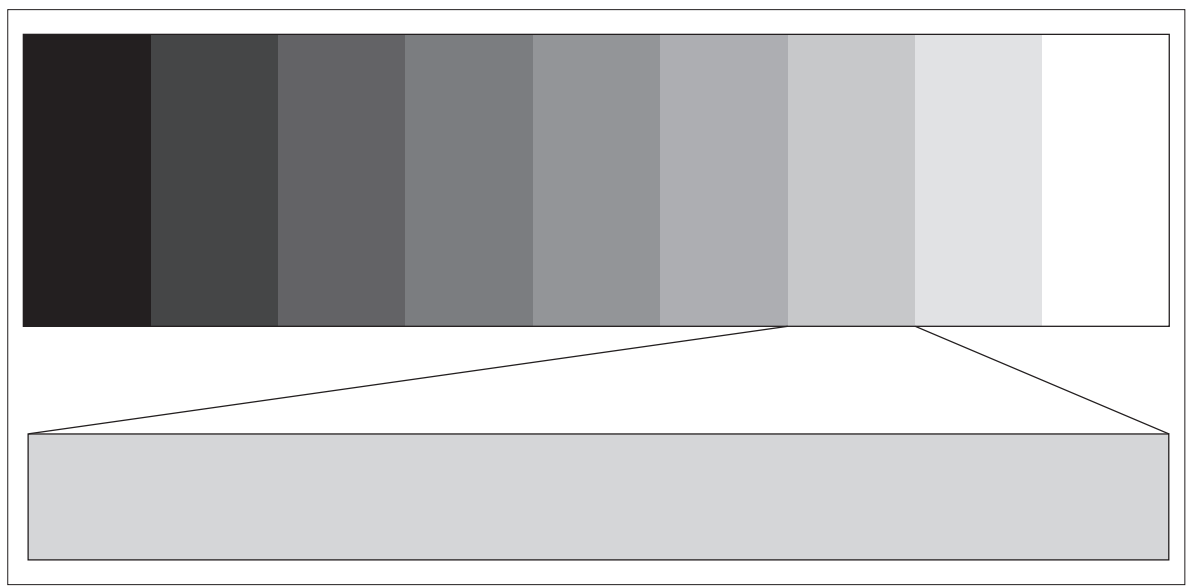

Fig. 6 Greyscale and 18\% grey or neutral density

colour temperature increases, the colour of the light changes from red (warm) to blue (cold).

\section{Natural daylight}

Daylight has a colour temperature of 6,540 K, which incorporates wavelengths of the bluish ultraviolet radiation. This type of illumination is useful for certain clinical applications (eg shade matching of ceramic restorations), as well as extra-oral photography (eg face, lips). The advantages of natural daylight are that it is economical (no purchase necessary!), readily available and 'what-you-see-iswhat-you-get' (WYSIWYG) in the resultant image. However, natural daylight is a misnomer; the light is warmer at sunrise, gets cooler at noon and warm again at sunset. Furthermore, even if the time is ideal, due to erratic weather conditions or shadows cast by buildings, foliage and animals, daylight is not a predictable light source. Finally, if light of an incorrect colour temperature is used, photographs have false colour rendition of either a red or blue colour cast.

\section{Electronic flash}

Two types of electronic flashes are available, compact (mounted onto the camera) and studio. Both varieties are commonly used as illuminations for dental photography. The light output of electronic flashes is corrected to 'photographic daylight' with a colour temperature of 5,500 K. Photographic daylight, as opposed to natural daylight, is ideal for photography 

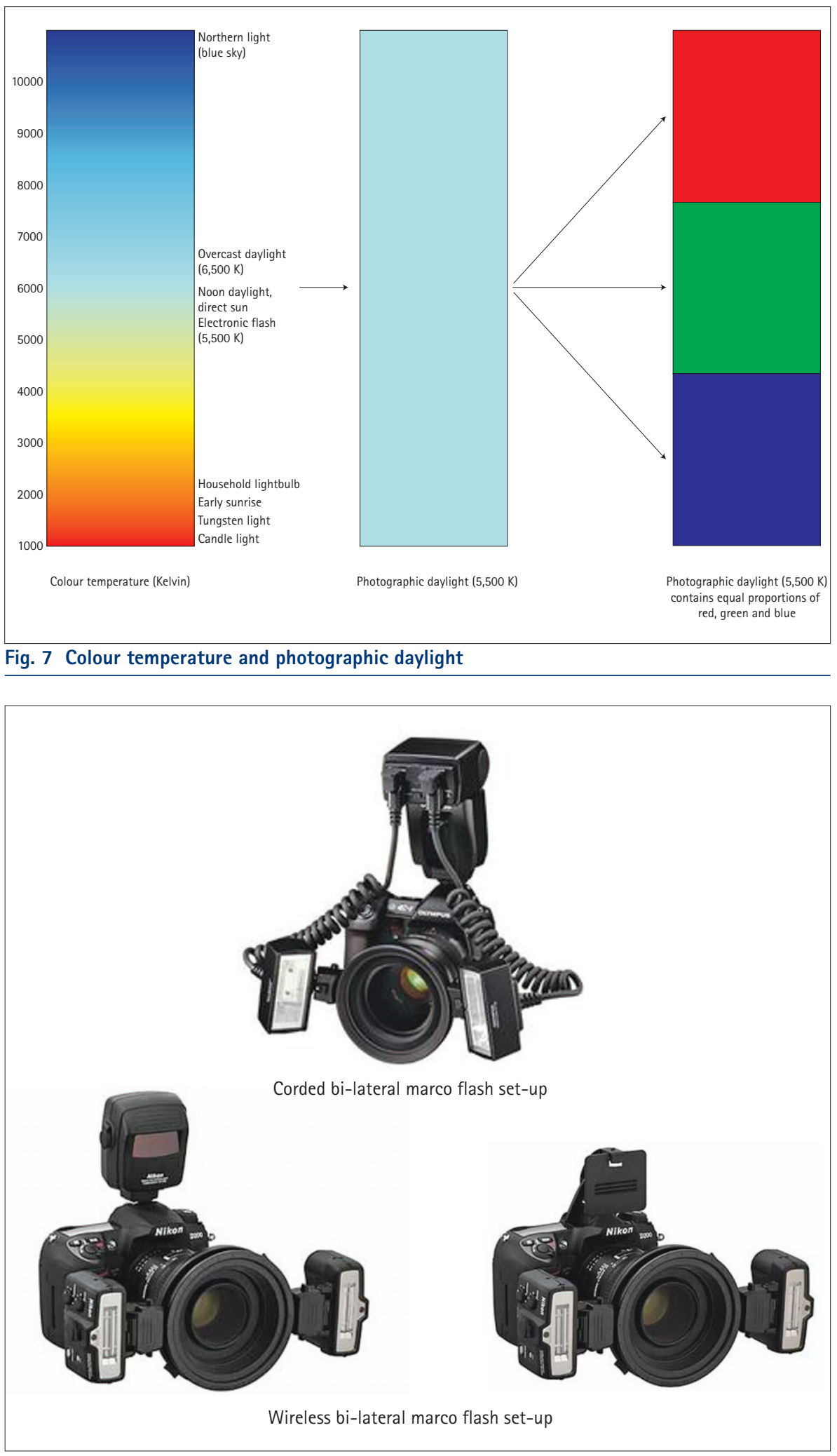

Fig. 8 A selection of uni-directional flashes mounted in a bi-lateral configuration

because it is only at this colour temperature that the three additive primary colours red, green and blue (RGB) are present in equal proportions (Fig. 7). Since all photography, including digital, uses the RGB model for image production, it is essential that the illumination contains each of these colours in equal proportions. The advantage of electronic flash is that the light output is predictable, instantaneous and universally adaptable for any type of camera. The major disadvantage is that WYSIWYG is not possible, and a photograph can only be scrutinised on the LCD camera viewer after taking the picture.

Camera mounted electronic flashes are

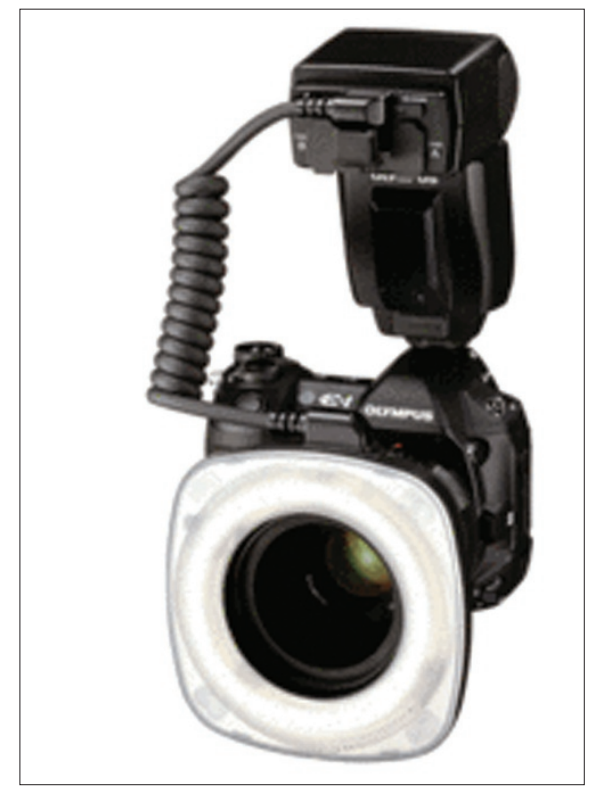

Fig. 9 A ring-flash for macro photography

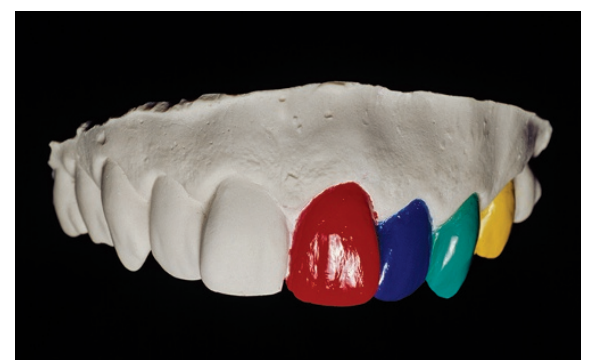

Fig. 10 Image photographed with a ring flash that is devoid of shadows, flat and bland

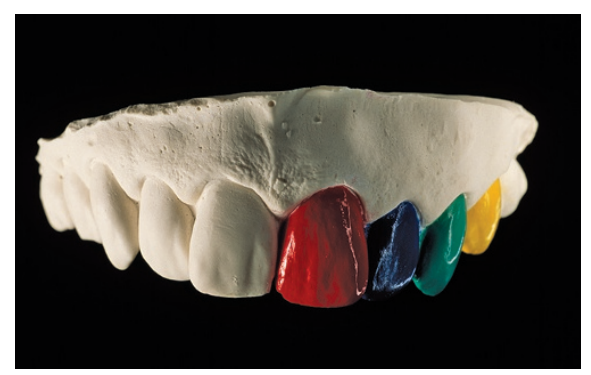

Fig. 11 Image photographed with unidirectional flashes mounted in a bi-lateral configuration. Notice shadows and highlight creating depth (compare with Figure 10)

available in numerous shapes and sizes, for example ring-flash or uni-directional (Figs 8-9). Ring-flashes create a uniform burst of light, useful for taking pictures of posterior teeth or areas of difficult access. The major drawback with ring flashes is the uniform light output, which creates a shadow-less, flat, bland and lacklustre image (Fig. 10). While excellent for illuminating posterior regions of the mouth, for anterior teeth or for restorations where aesthetics are of paramount concern, ring flashes are not recommended since the uniform burst of light obliterates fine detail, 
translucency and subtle colour transitions within individual teeth.

On the other hand, uni-directional flashes create shadows and highlights and the teeth and gingivae appear more three dimensional, with increased contrast and detail (Fig. 11). The usual directional lighting for dental photography is two bi-lateral flashes mounted on a bracket. The bracket allows the flashes to be positioned as desired, depending on the shape of the patient's arch form and tooth alignment. This set-up is the ideal choice when photographing anterior teeth for capturing nuances of characterisation and colour, texture and translucency, which are critical for aesthetic anterior prostheses. Uni-directional illumination also offers versatility to highlight specific details within a tooth, which guides the ceramist to mimic these characterisations in the final prosthesis.

\section{Principle of the angle of incidence}

Dental photography presents a unique photographic scenario: not only does a photograph need to record the highly reflective enamel surface, but also a layer below it (dentine).

When highly reflective surfaces are photographed, the lighting set-up should ensure that unwanted halation is avoided. This is achieved by exploiting the principle of the angle of incidence. If a single light source is used to photograph glass, its reflection will cause a 'hot spot' or specular reflection termed halation. While this is useful for capturing surface texture, it can be annoying for visualising objects behind the glass (analogous to visualising dentine strata below enamel).

In order to mitigate unwanted reflections, two light sources are used, positioned on both sides of the camera at $45^{\circ}$ in the horizontal plane (bi-lateral set-up). With this twin light set-up, the angle of incident from one flash equals the angle of reflectance of the contra lateral flash. The result is that reflections from the two light sources cancel each other out, and hence the camera does not 'see' any reflections. This type of lighting can be used to photograph dentine strata below the overlying enamel of natural teeth, or to visualise the dentine porcelain layer of a ceramic dental restoration.

Electronic flashes are usually specific to a particular DSLR and are not interchangeable with cameras of another manufacturer. An important feature to look for when purchasing these flashes is to ensure that they are detachable from the camera, preferably with wireless connections. This is particularly useful for extra-oral images in the dental laboratory (discussed further in Part 7). If this facility is not possible with camera specific flashes, the other option is to purchase third party electronic flashes such as the range from Metz (www.metz.de). These flashes, combined with a manufacturer specific adaptor, can be used with any camera and offer greater latitude for taking other types of pictures such as bench shots in a dental laboratory.

\section{Tungsten and quartz lighting}

Tungsten light is warmer (reddish) with a colour temperature of 3,400 K (Fig. 12). Prior to the advent of electronic studio flashes, tungsten photoflood lamps were the lights of choice for all studio set-ups. The advantages are WYSIWYG, they are relatively inexpensive, portable, and useful for indoor photography since the colour temperature matches most domestic lighting. Tungsten lights are particularly useful for photographing plaster casts of teeth and dental prostheses, but excessive heat generation from the lamps makes their use for clinical applications impractical.

\section{Continuous photographic daylight}

HMI illumination has a similar output to electronic flashes, but rather than a single burst, the light is continuous. These lamps are capable of delivering light at a constant 5,500 K colour temperature, and are mainly used for the motion picture industry. The obvious advantage is WYSIWYG, but presently their exorbitant cost makes them prohibitive for dental use.

\section{LED}

LED (light emitting diode) is a relatively new type of illumination for dental photography. Various models and configurations are available which are attached to the front of the camera lenses, for example Optilume (Optident, UK). The advantages are WYSIWYG and constant colour temperature of 5,500 K, but the major drawback is the low intensity illumination, which requires large apertures for correct exposure that severely reduces the depth of

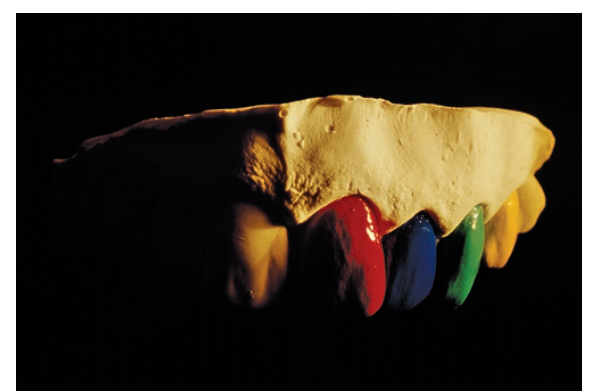

Fig. 12 Image photographed with tungsten light with a colour temperature of around $3,400 \mathrm{~K}$

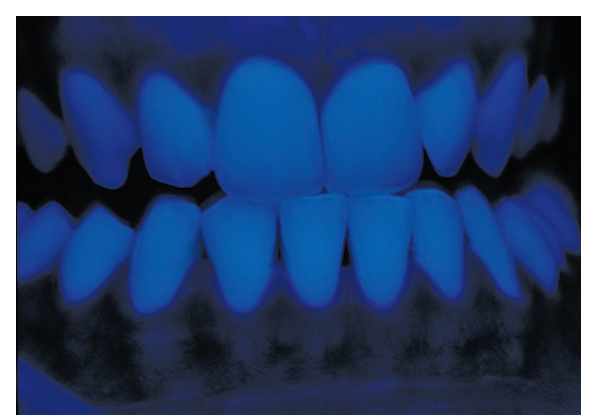

Fig. 13 UV light reveals intrinsic fluorescence of natural teeth

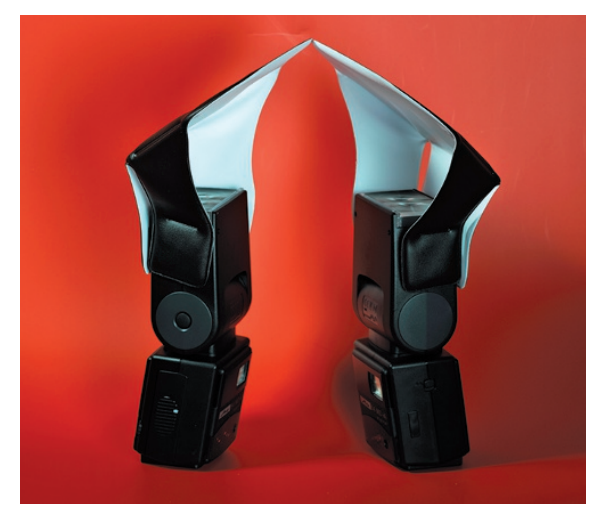

Fig. 14 Diffusers help mute the flash output, giving a softer lighting

field. Alternately, rather than compromising the depth of field, the shutter speed can be prolonged but this produces unwanted camera shake and blurred images. Another disadvantage is LED light sources require a relatively large battery pack, which is cumbersome and annoying.

\section{UV}

UV (ultraviolet) illumination is useful for showing intrinsic fluorescent properties of natural teeth (Fig. 13), or for checking imperfections within ceramic restorations such as fractures or porosity. However, caution is necessary when using ultraviolet lights to photograph restorations in the mouth. Some UV light sources have deleterious effects on the eyes and should never be used. Even 


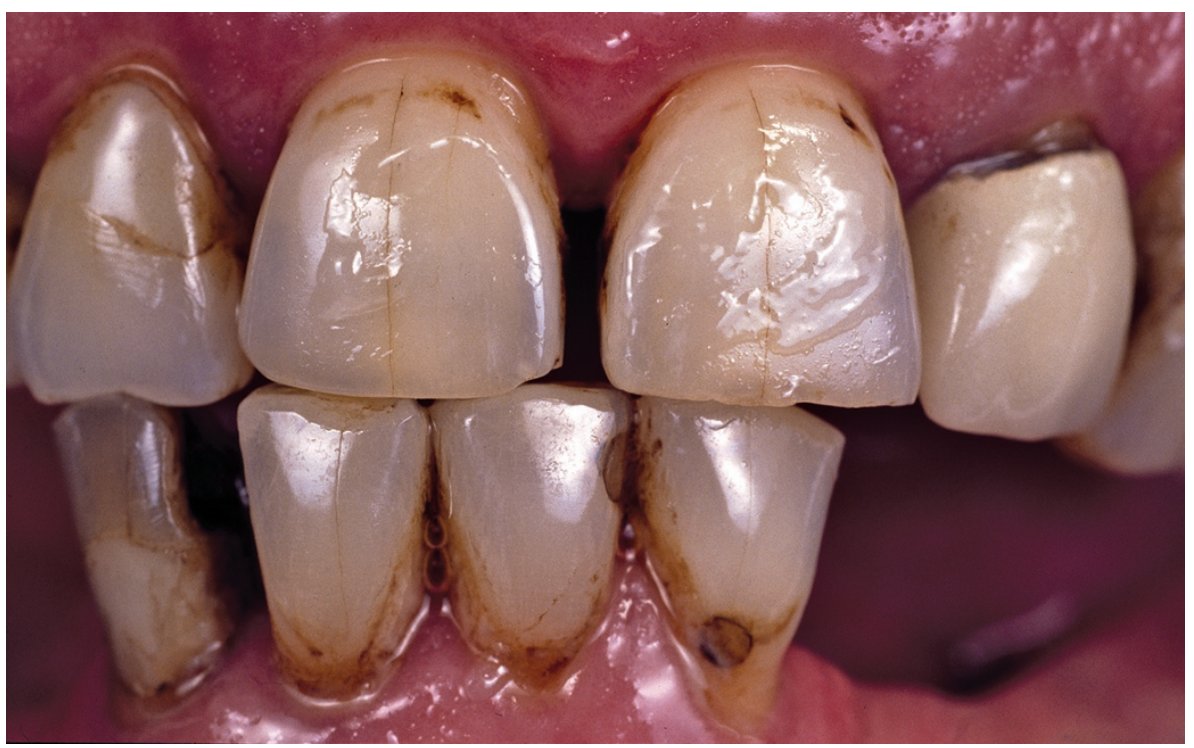

Fig. 15 Photograph using naked flashes, which capture surface enamel texture. Notice the saliva flowing off the enamel surface

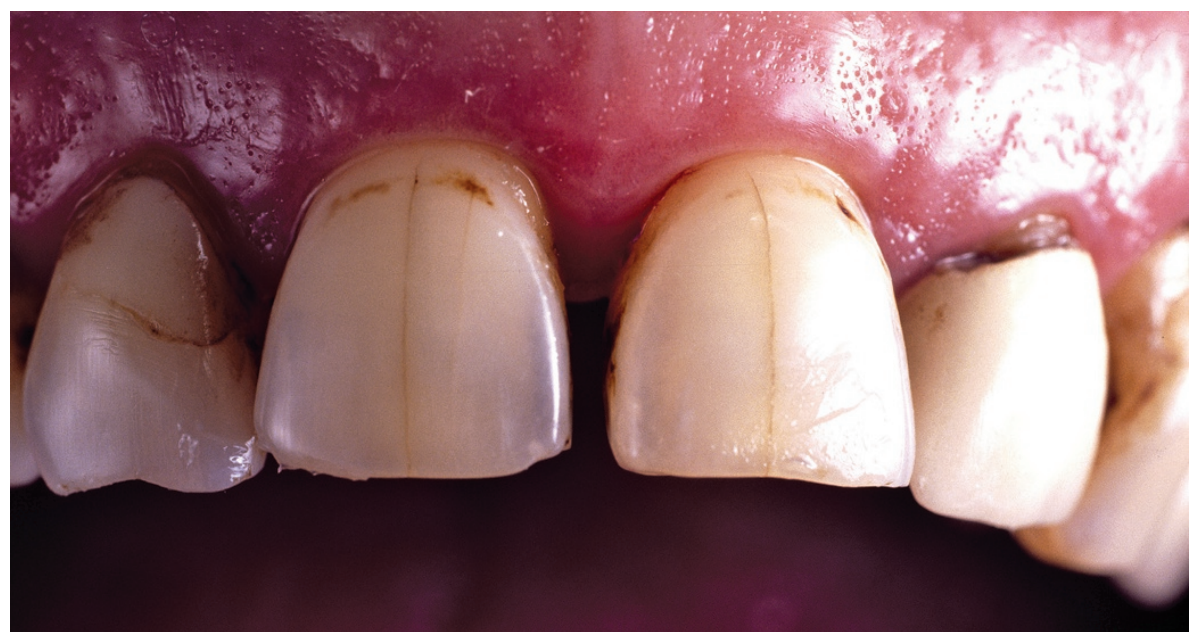

Fig. 16 One flash and a silver reflector set-up allows light to pass through the enamel layer for revealing enamel cracks and dentine strata characterisations

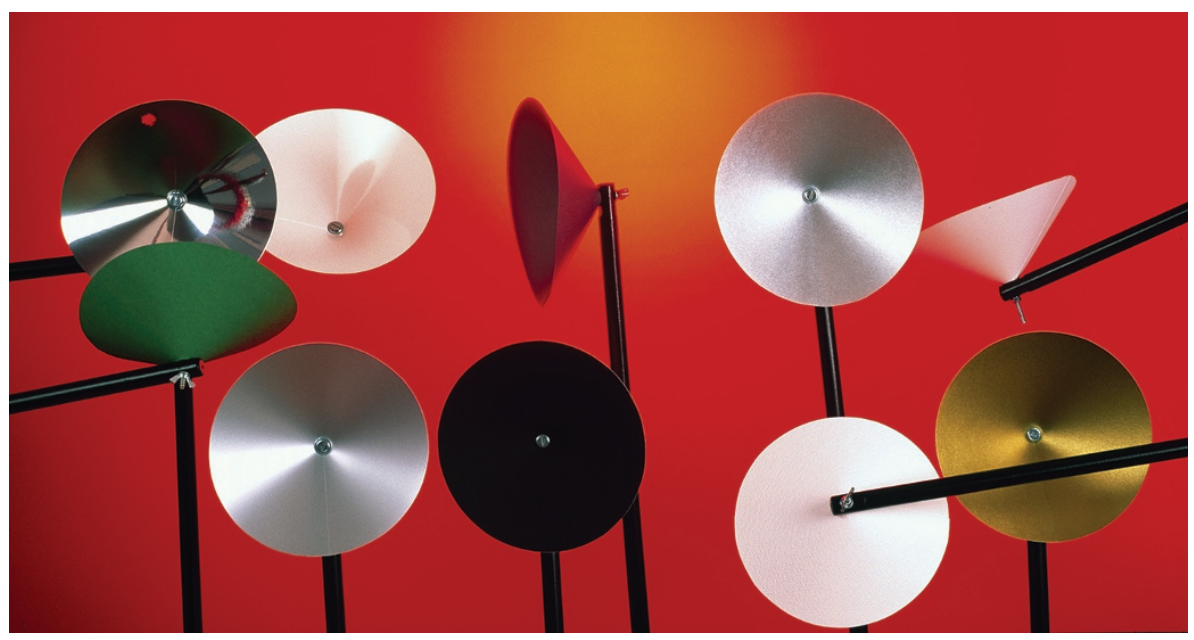

Fig. 17 Custom made reflectors for use in dental photography

with safer varieties of UV lights, the patient and all personnel in the room must wear special UV protection glasses.

\section{MANIPULATING LIGHT}

The next stage after choosing an illumination is discussing methods to modify the light source. Regardless of the colour temperature, light needs to be modified depending on the application or assignment. For example, the lighting set-up to capture margins of a prepared tooth differs from that needed to reveal dentine strata beneath the enamel layer. In each situation a specific lighting set-up is necessary to record the salient information of an object.

\section{Angle of illumination}

Illumination for dental photography can either be uniform or directional. Uniform illumination is $360^{\circ}$ coverage, typically produced by an electronic ringflash or directional lighting, which are discussed above.

\section{Modifying light}

In addition to position, flashes also require muting or accentuating for recording different aspects of the dentition. For example, to show enamel or crown texture, the lighting set-up is two bi-laterally positioned naked flashes to create specular reflections off the surface with enhanced contrast. A light source can be modified by a variety of methods, which are categorised as follows:

1. Naked light - no modification: harsh output, primarily used for high contrast images, for capturing texture and lustre of enamel and porcelain surfaces

2. Blocking light - for example, by a card, eliminates the light from the set-up. This is useful in a bi-lateral twin flash set-up, where unilateral illumination is required for creating shadows on the opposite side of the main flash. This is also a useful method for photographing plaster casts in the dental laboratory (to be covered in Part 7)

3. Diffusion - by placing materials (cloth or Perspex) of varying opacities in front of the light source. This has two effects (Fig. 14): firstly the output is reduced, and secondly the emitted light is softer. The larger the diffuser, the softer the output. The softer emission is ideal for creating subtler images, but with reduced contrast

4. Reflection - with a reflective card placed opposite a naked or diffused light source. The resulting light 
depends on the surface used to reflect the light. Reflected light is especially useful for eliminating unwanted halation or highlights from highly reflective surfaces and is an excellent method for documenting dentine shade and characterisations (Figs 15-16). For dental purposes reflectors are limited to only a few types, usually art card with different reflective surfaces which can be cut to size and disposed of after a single use. Also, in the dental laboratory, custom-made reflectors (Fig. 17) are invaluable for photographing casts, wax-ups and transparent stents (to be covered in Part 7).

\section{Coloured reflectors}

A highly polished glossy surface, irrespective of its colour, produces a specular (mirror-like) reflection. Conversely, a textured or matt coloured surface creates reflections that correspond to its colour. For example, a glossy green card will produce a reflection that is specular, while a matt green card will produce a reflection with a greenish hue. The latter type of coloured reflection, if judicially exploited, can be used to shift the colour balance of an image. However, caution is necessary: coloured reflections may yield a pseudocolour rendition. For example, a clinician's green surgical gown can cast an unwanted greenish tint onto the enamel surfaces of teeth.

\section{Mirror reflectors}

A highly polished mirror or mirror-like card behaves similarly to a glossy surface mentioned above and produces a similar light output.

\section{Silver reflectors}

A silver card reflector produces a muted output that increases the overall colour temperature, that is, more blue, and tends to flood the scene with a burst of bright light. Most floodlights in theatres employ

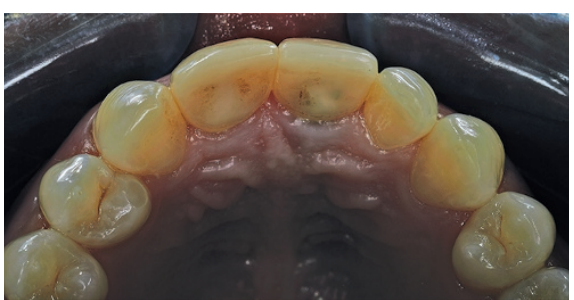

Fig. 18 An underexposed image with an intra-oral mirror

these types of metallic reflectors. If the texture of the silver card is increased (ie made rougher), the light is diffused further. The effects on the image are softer shadows and reduced contrast. This type of light is ideal for portraiture to soften or eliminate unflattering facial blemishes and wrinkles (covered in Part 7). The disadvantage is that texture is indiscernible, yielding a flatter image that is less vibrant compared with a polished reflector.

\section{Gold reflectors}

Gold coloured reflectors are identical to silver, their analogues, but the overall colour temperature is warmer, creating a subdued romantic ambience.

\section{White reflectors}

White surfaces produce the most diffuse type of reflection. The reflected emissions are extremely soft, much more subtle and less specular compared to mirror, silver or gold reflectors. The resulting light does not 'flood' the image, but subtly reduces shadows. Once again the degree of texture influences the extent of diffusion.

\section{Grey reflectors}

While all the above reflectors tend to 'reflect' the majority of light falling on their surfaces, the reverse is true of grey cards. Depending on the intensity of grey, the majority of the light is absorbed and a minority reflected. As mentioned previously, grey cards are primarily used to ascertain exposure (18\% photographic grey card) and calibration of the white balance of digital images (to be covered in Part 6). Nevertheless, in rare situations they may be useful for partial elimination of shadows.

\section{Other factors for modifying light}

The power output obviously influences the intensity of light. Some compact electronic flashes allow output intensities to be altered. However, greater latitude is possible with studio lights where output can be increased or reduced depending on the prevailing set-up.

Flash-to-subject distance also influences light intensity, which depends on the inverse square law. Simply stated, illumination is less bright the further away from its source because it has a greater area to cover. For example, it the distance is doubled, the light not only has to travel twice the distance, but also has to cover a larger area. The implication of the inverse square law is that if the exposure is f22 and the distance is doubled, an increase of two, not one f stops is necessary for the new exposure, that is, f8. The opposite is true if the distance is halved; the correct exposure would then decrease by two f stops, that is, f64. The inverse square law is applicable when taking intra-oral images using mirrors. In these circumstances the light from the flashes has to travel a greater distance by being reflected off the mirror surface before it can illuminate the teeth. Exposure compensation is therefore necessary to avoid under-exposed images (Fig. 18).

Optical supplementary attachments such as magnifying lenses can be attached to studio lights for focusing the light beam from as small as $1^{\circ}$ to about $10^{\circ}$, which allows very precise coverage as small as $1 \mathrm{~mm}^{2}$. Other attachments utilise fibreoptic cables for 'bending' light, thereby increasing access to unlit recesses in a photographic composition. Fibre-optic cables can also be used to trans-illuminate all-ceramic dental restorations to show porcelain layering or for scrutinising imperfections within the ceramic material (to be covered in Part 7). 